\title{
Carnets
}

Revue électronique d'études françaises de l'APEF

Deuxième série - 4 | 2015

Regards sur Camus

\section{Un héros de leur temps}

\section{Vlasta Dufková}

\section{(2) OpenEdition \\ Journals}

Édition électronique

URL : http://journals.openedition.org/carnets/1526

DOI : 10.4000/carnets. 1526

ISSN : 1646-7698

Éditeur

APEF

\section{Référence électronique}

Vlasta Dufková, "Un héros de leur temps », Carnets [En ligne], Deuxième série - 4 | 2015, mis en ligne le 30 mai 2015, consulté le 19 avril 2019. URL : http://journals.openedition.org/carnets/1526 ; DOI :

$10.4000 /$ carnets. 1526

Ce document a été généré automatiquement le 19 avril 2019

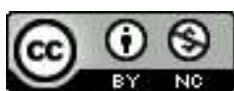

Carnets est mis à disposition selon les termes de la licence Creative Commons - Atribution - Pas d'utilisation commerciale 4.0 International. 


\section{Un héros de leur temps}

\section{Vlasta Dufková}

1 En traductrice tchèque des Carnets d'Albert Camus j'ai pu, lors du colloque international Camus, de l'autre côté du «Mur». Réceptions de l'œuvre camusienne en Europe médiane qui s'est déroulé à Paris le 31 mai 2013, proposer une hypothèse basée sur ma lecture interprétative d'un passage du troisième tome des Carnets, en rapport possible avec le sujet du dernier grand texte achevé de Camus. Et, faute d'article publié à ce propos ${ }^{1}$, il me faudra commencer par exposer en raccourci cette supposition avant de développer une réflexion plus large sur un/le héros des années cinquante du $\mathrm{xx}^{\mathrm{e}}$ siècle - celui de La chute.

\section{Liberté, fraternité}

2 En 2000, au moment de traduire l'Appendice au Cahier $n^{\circ}$ VIII des Carnets, datés d'août 1954 à juillet 1958, je me suis heurtée à un nom énigmatique : « Kalande ». Celui-ci figure dans un poème sans titre et signé « Pierre Serment » qui, visiblement, est le brouillon d'un pastiche amer du célèbre "Liberté » de Paul Éluard et traite de la trahison de cette liberté mythique, chantée par le poète de la Résistance :

De l'encre des prisons

sur les chaînes de l'esclave

au doux visage des fusillés

j'écris ton nom

Liberté

Tes jambages sont des barreaux

ton visage est un verrou

fraternel aux bourreaux

Sur les ordres des guichets

J'écris ton nom

Liberté

Liberté, liberté trahie

Où sont tes défenseurs?

Dans la nuit des caves 


\author{
Tes doux yeux ont crépité \\ J'écris ton nom \\ Kalande $^{2}$ meurt \\ Facile est écrire \\ terrible est mourir \\ J'écris, j'écris \\ J'écris ton nom adultère \\ Sur le tien qui désespère \\ Oh! Qu'as-tu fait de ma jeune \\ Kalande $^{3}$ ? On meurt nu \\ Quand vos frères vous tuent \\ J'écris ton nom sonore \\ D'une encre qui déshonore \\ Pour barrer l'avenir \\ Pour raturer le souvenir \\ J'écris ton nom \\ Liberté \\ En capitales de douleur
}

Pierre Serment (Camus, 1989 : 245-246)

Par le dernier vers, le pasticheur au nom significatif souligne, par une allusion ouverte au titre du premier recueil du poète ${ }^{4}$, "en capitales de douleur» le nom de Liberté, obstinément réécrit et apostrophé le long du poème dans une sorte de refrain éluardien. Déjà les épithètes toutefois - « liberté trahie », « nom adultère », « encre qui déshonore ${ }^{5}$ »signalent un radical changement de contexte, lié à la mort de "ma jeune Kalande » dont la responsabilité incombe à des «frères " puisque l' "On meurt nu / Quand vos frères vous tuent ». De plus, la grandeur de l'écriture, qui se veut vivifiante et régénératrice chez Éluard ${ }^{6}$, est ici diminuée vis-à-vis de l'horreur de mourir: «Facile est écrire / terrible est mourir $"$.

\title{
Cécité
}

Impossible pour moi de ne pas voir la proximité du nom Kalande qui tient la clé de lecture du poème avec celui d'un intellectuel de gauche tchèque : Záviš Kalandra (1902-1950), exmembre du groupe surréaliste pragois, historien et éminent journaliste communiste avant d'être exclu du Parti, en 1936, pour avoir dénoncé ouvertement et à plusieurs reprises les procès politiques de Moscou. Revenu en Tchécoslovaquie en 1945 après six années de déportation nazie, il est arrêté à nouveau en 1949, un an et demi après le coup d'État communiste, puis exécuté, le 27 juin 1950, suite à un simulacre de procès où il a récité des aveux absurdes, appris par cœur (Doležalová, 2008-2013).

Dix jours avant cette exécution, Albert Camus signe, avec une cinquantaine d'intellectuels français, un télégramme envoyé au Président de la République Tchécoslovaque et présentant un recours en grâce ${ }^{7}$. Le télégramme fait suite à la lettre ouverte d'André Breton ${ }^{8}$ publiée dans le Combat du 13 juin 1950 : Breton, qui connaît et apprécie Kalandra depuis sa visite à Prague avec Paul Éluard, en 1935, s'engage particulièrement pour sauver de la peine de mort leur ami commun et un ancien du surréalisme. Éluard, toutefois, en tant que membre du Parti communiste français, refuse d'intervenir en sa faveur et 
décline l'appel que Breton lui a adressé, par les mots devenus tristement célèbres : «J'ai trop à faire avec les innocents qui clament leur innocence pour m'occuper des coupables qui clament leur culpabilité ${ }^{~ » . ~ E n ~ 1951, ~ d a n s ~ L ' ~ H o m m e ~ r e ́ v o l t e ́, ~ C a m u s ~ f e r a ~ a l l u s i o n ~ a ̀ ~ c e t t e ~}$ sorte de naïveté ou aveuglement idéologique de Paul Éluard, incapable d' imaginer « que son ami Kalandra dût mourir » de cette "vie véritable, comme l' amour » qu'était la révolution, conçue comme " un mythe absolu et consolateur ${ }^{10}$ " (Camus, $1962: 123$ ).

Voilà les faits historiques qui me font supposer l'éventualité d'un petit mot mal lu dans le manuscrit du dernier volume des Carnets, celui de «ma » au lieu de «mon» qui, devant l'adjectif « jeune » et suivi de près par « Kalandra » au lieu de "Kalande », changerait de sexe le mystérieux personnage. On croirait presque tenir la clé de l'énigme, cependant la question du genre n'est pas cruciale, car en principe il s'agit toujours de Kalandra, que son nom soit donné dans sa version correcte, ou modifié et mis au féminin.

\section{Amitié}

7 Peu de temps après, en 1952, Paul Celan, le grand poète de langue allemande vivant à Paris, écrit un "In memoriam Paul Éluard", après le décès inopiné de son confrère français :

\begin{tabular}{|c|c|}
\hline Paul Celan & Traduction de Valérie Briet \\
\hline IN MEMORIAM PAUL ELUARD & IN MEMORIAM PAUL ELUARD \\
\hline $\begin{array}{l}\text { Lege dem Toten die Worte ins Grab, } \\
\text { die er sprach, um zu leben. } \\
\text { Bette sein Haupt zwischen sie, } \\
\text { laß ihn fühlen } \\
\text { die Zungen der Sehnsucht, } \\
\text { die Zangen. }\end{array}$ & $\begin{array}{l}\text { Pose les mots dans la tombe pour le mort, } \\
\text { ceux qu'il a dits pour vivre. } \\
\text { Couche sa tête entre eux, } \\
\text { fais-lui sentir } \\
\text { les langues de la nostalgie, } \\
\text { les sangles. }\end{array}$ \\
\hline $\begin{array}{l}\text { Leg auf die Lider des Toten das Wort, } \\
\text { das er jenem verweigert, } \\
\text { der du zu ihm sagte, } \\
\text { das Wort } \\
\text { an dem das Blut seines Herzens } \\
\text { [vorbeisprang, } \\
\text { als eine Hand, so nackt } \\
\text { wie die seine, } \\
\text { jenen, der du zu ihm sagte, } \\
\text { in die Bäume der Zukunft knüpfte. }\end{array}$ & $\begin{array}{l}\text { Pose sur les paupières du mort le mot } \\
\text { qu'il a refusé à celui } \\
\text { qui lui disait tu, } \\
\text { le mot } \\
\text { que le sang de son cœur a passé } \\
\text { alors qu'une main, nue } \\
\text { comme la sienne, } \\
\text { l'a pendu, celui qui lui disait tu, } \\
\text { aux arbres de l'avenir. }\end{array}$ \\
\hline $\begin{array}{l}\text { Leg ihm dies Wort auf die Lider: } \\
\text { vielleicht } \\
\text { tritt in sein Aug, das noch blau ist, } \\
\text { eine zweite, fremdere Bläue, } \\
\text { und jener, der du zu ihm sagte, } \\
\text { träumt mit ihm : Wir. }\end{array}$ & $\begin{array}{l}\text { Pose ce mot-là sur ses paupières: } \\
\text { peut-être } \\
\text { que dans son œil encore bleu } \\
\text { pénètre un second bleu, plus étranger, } \\
\text { et celui qui lui disait tu } \\
\text { rêve avec lui : Nous. }\end{array}$ \\
\hline
\end{tabular}


(Celan, $1991: 94-95)$

Le poème se réfère à la même situation du couple d'amis Éluard-Kalandra, en relation avec le poème emblématique d'Éluard, bien que le mot vivifiant de «Liberté » y soit laissé en creux, tout comme le nom de l'ami supplicié : il est évident que le triste épisode n'est pas passé inaperçu. Deux grandes décennies plus tard, il résonnera encore dans Le Livre du rire et de l'oubli de Milan Kundera, publié en 1978, où l'auteur tchèque décrit à sa façon la cécité idéologique d'Éluard, qui l'avait personnellement marqué ${ }^{11}$.

\section{Clamence}

9 Essayons de placer le poème inclu dans les Carnets dans le contexte de l'œuvre camusienne : il est à peu près sûr que la parodie amère de "Liberté » fut écrite à la même époque que La Chute, paru en 1956. Et à regarder de près ce récit ambigu et à maintes facettes, on trouvera bien des traits rapprochant les deux textes apparemment hétérogènes: à commencer par «Pierre, vous savez, le froussard, Pierre " (Camus, 1997 : 122), l' apôtre ayant renié le Christ qui, avec un « sens d'humour » incontestable et " poussant l'ironie » à bout a déclaré bâtir " sur cette pierre » son église (ibid.) : cet apôtre que l' on reconnaît sans peine dans l' auteur prétendu du poème. Le "dernier cercle " (Camus, 1997 : 18) de l'Enfer dantesque, évoqué dès le début du récit de Clamence, « [1]e cercle des... » est celui des traitres innommés et pouvant faire penser au Serment, mais c'est aussi «le lieu de la chute de Lucifer » (Lévi-Valensi, 1996 : 128), l'ange déchu. Une autre allusion à Dante évoque les Limbes, « une sorte de vestibule de son enfer » (Camus, 1997 : 89), auquel Clamence compare le monde humain, où nous sommes tous coupables : « Nous n'avons ni l'énergie du mal, ni celle du bien. (...) Nous sommes dans le vestibule » ( ibid.) - l'endroit dantesque "appelé 'le vestibule des lâches ${ }^{12}$ ' par certains commentateurs » (Lévi-Valensi, 1996 : 129).

10 Étant donné la fameuse réponse d'éluard, qui devait résonner dans la mémoire de Camus et où le verbe "clamer " était relié aussi bien à l'innocence qu'à la culpabilité, il ne paraîtra pas trop osé d'élargir le champ symbolique du nom de «Clamence ", déjà riche, bien que la relation entre les deux ne soit sûrement pas toute directe. Sous cette optique, le thème récurrent d'amitié dépassera lui aussi le contexte des seuls Temps modernes sartriens et la métaphore de l' " homme dont l' ami avait été emprisonné et qui couchait tous les soirs sur le sol de sa chambre pour ne pas jouir d' un confort ${ }^{13}$ qu' on avait retiré à celui qu' il aimait» (Camus, 1997:36) se teintera de nouvelles associations concrètes, surtout par opposition au malconfort, supplice moyenâgeux, évoquant immédiatement les méthodes staliniennes de culpabilisation des innocents.

11 De même, dans la grande tirade énumérative à la première personne sur la «liberté » toujours « à la bouche » (Camus, 1997 :138), on peut voir, assurément, un témoignage à charge dans le procès contre Sartre et les existentialistes parisiens, donnant raison à l'interprétation de La chute qui prend ces derniers pour la principale sinon l'unique cible du persiflage. Ici aussi, néanmoins, il suffira d'un léger changement d' optique pour faire resortir sous la prose une structure de vers potentiels et pour voir et/ou entendre dans la forme de ce qui est dit aussi bien un écho lointain et négatif du poème emblématique dont le «maître mot», tu chez Éluard, pudiquement, jusqu'au dernier vers, est ici complètement déprécié : 
Je l'étendais au petit déjeuner sur mes tartines,

je la mastiquais toute la journée,

je portais dans le monde une haleine délicieusement rafraîchie à la liberté.

J'assenais ce maître mot à quiconque me contredisait,

je l'avais mis au service de mes désirs et de ma puissance.

Je le murmurais au lit,

dans l'oreille endormie de mes compagnes,

et il m'aidait à les planter là.

Je le glissais... (Camus, $1997: 138)^{14}$

12 Le traitement de la liberté - et de la langue - n'étant pas réservé aux seuls philosophes, la responsabilité qui en découle concerne aussi les poètes.

\section{Jeune Kalande}

13 Rien n'est définitif dans ce texte à l'omniprésente dialectique vérité-mensonge, impossible à démêler, car « [o]n voit parfois plus clair dans celui qui ment que dans celui qui dit vrai » (Camus, 1997:126). Sans oublier l' évidente théâtralisation du texte, où l' ironie du monologue auto-accusateur du juge-pénitent fait penser à celle, beaucoup plus cuisante et tragique, dont témoigne le document filmé de l'aveu mémorisé du prétendu «trotskiste » Záviš Kalandra lors de son procès-spectacle. La tragédie de sa "performance » est double : non seulement il joue le jeu imposé par le «malconfort » stalinien en répétant le rôle mémorisé mais encore il croit jouer son jeu à lui puisqu' il ne se défend que par l'évidente ironie de la diction, profondément convaincu que pour ses amis, elle fera ressortir l'évidente absurdité de ses propos. La réaction de Paul Éluard prouve, entre autres, qu'on ne manie pas le mensonge impunément, même dans des conditions aussi absurdes, et que le masque est déjà une culpabilité. Kalandra, un Lorenzaccio sans meurtre, est aussi Záviš K., le protagoniste de son Procès à une époque sans innocents, car sans Loi. D'une manière pervertie, lui aussi est Clamence ou, plus précisément, il prépare sans le vouloir le sol à toute sorte de Clamences des temps modernes.

Qui d'autre que lui aurait davantage le droit de dire : «Plus je m'accuse et plus j'ai le droit de vous juger » (Camus, 1997 : 146) ? Lui, qui dès avant la guerre avait prévu le futur - y compris le sien - avec une bonne dose d' humour noir qui nous glace, pour ne citer qu' un de ses collages, un vrai chef-d'œuvre en matière de "poisson d'avril " humoristique, paru dans le $n^{\circ} 13 / 1937$ de la revue Světozor. Kalandra y avait utilisé sa propre photographie : assis sur une chaise dans la partie droite du tableau et interrogé par la Guépéou stalinienne (précurseur de la NKVD, devenue plus tard la KGB), dans un décor de bureau minable qui met en valeur, ironiquement, une grande peinture du $\mathrm{XIX}^{\mathrm{e}}$ siècle représentant la mort de Přemysl Otakar II, il avoue avoir assassiné ledit roi de Bohême lors de la bataille de Marchfeld, en $1278 .{ }^{15}$ Plutôt que surréaliste, l'image évoque l'atmosphère du Brave soldat Chvéik ${ }^{16}$ de Jaroslav Hašek, quoique teintée, dans la perspective historique, du Procès de Kafka. La légende du collage ${ }^{17}$, entretissée de métaphores propagandistes, que l'on a pu entendre lors des procès de Moscou et, plus tard, de ceux de Prague, finit par annoncer que «le procureur général transmettra le texte détaillé de la sentence au tribunal public !»

Il serait exagéré d'imputer à Camus la connaissance des choses qu'à l'époque il ne pouvait qu'entrevoir. Cependant, si l'on reconnaît dans les romans de Kafka une vision des choses imminentes, du même droit peut-on également lire dans La Chute un ou plusieurs traits 
qui se font jour à travers l'énigmatique «jeune Kalande ». On pourrait même donner ce joli nom à la jeune fille noyée, apostrophée à la fin de La chute : « Ô jeune Kalande, jette-toi encore dans l'eau pour que j'aie une seconde fois la chance de nous sauver tous les deux!»

Une fois l' «imprudence» (Camus, $1997: 153)$ d'exprimer ce désir commise, une ironie quasi «christique » - ou diabolique - fait dire au petit prophète Clamence : «Il faudrait s'exécuter $^{18}$. Brr... ! l'eau est si froide ! » (ibid.).

Sinon, l’unique exécuté serait-il Kalandra?

\section{Engagement}

18 La question est moins extravagante qu'elle ne pourrait paraître. Si la chute du corps de la suicidée dans la Seine et son cri qui hante le narrateur resté inactif - en dehors de l' improbabilité d' un secours efficace - peuvent évoquer les tendances suicidaires de la femme dépressive de Camus, ou bien représenter la situation modèle de la lâche nonassistance à autrui, si illusoire qu'une assistance puisse être, le sens métaphorique de la scène va jusqu'à désigner l'engagement civique de l'écrivain et intellectuel. Le «monologue dramatique» ou "dialogue implicite», pour me servir des termes de la présentation enrichissante de La chute par Jacqueline Lévi-Valensi, d' où j'ai aussi puisé ses successifs titres provisoires - Le Jugement dernier, Le Pilori, Bon apôtre, Un puritain de notre temps, Le Cri et Un héros de notre temps - est, en tout cas, bien ancré dans la « terrible époque ", tout en renvoyant à la condition humaine, celle de l'homme absurde.

Au stade d'Un héros de notre temps, calqué sur le titre du roman de Mikhail Iourievitch Lermontov, de 1841, la "version 4 [des cinq versions successives de La chute] emprunte quelques lignes à Lermontov» (Lévi-Valensi, 1997 : 33), et cette caractéristique par le sosie russe d'un enfant du siècle sera reprise en épigraphe de la traduction anglaise de $L a$ chute (ibid.) : «Un héros de notre temps est effectivement un portrait, mais ce n'est pas celui d'un homme. C'est l'assemblage des défauts de notre génération dans toute la plénitude de leur développement $»$.

20 Néanmoins, ceux que le juge-pénitent Clamence appelle «nous autres, enfants du demisiècle» (Camus, 1997: 130) sont confrontés à la responsabilité de l'immédiat aprèsguerre, où l'on décide de la vie et de la mort des autres, non seulement dans un ailleurs temporel ou spatial, sous des régimes totalitaires, mais aussi « ici et maintenant », vis-àvis des compatriotes ayant collaboré avec l'ennemi, sans parler de la guerre d'Algérie qui suivra de près. À croire l' article étoffé sur Wikipédia dont je me suis contentée à ce stade de la réflexion, on estime entre 10000 et 11000 morts le total de l' épuration effectuée à la Libération de la France, dont 8775 exécutions sommaires lors de "l'épuration extrajudiciaire avant le débarquement de Normandie »: le reste, soit plus d' un mille et demi, sont des condamnés à mort par les cours de justice et les cours martiales (Wikipédia, 2014).

21 Après la condamnation de l' écrivain Robert Brasillach, qui sera fusillé le 6 février 1945, la pétition de soutien au recours en grâce divise la communauté littéraire : bien que Camus, avec Éluard et Sartre, entre autres, appartienne au clan des « intransigeants », formé par la nouvelle génération née de la Résistance et revendiquant la pleine responsabilité de l' écrivain, lui finit par joindre sa signature à celles de ses aînés « indulgents » (ibid.) pour 
tenter de sauver la vie de l' homme, tout en gardant l' intransigeance dans le mépris manifeste à l' égard de sa conduite.

Le « demi-siècle » est visiblement une époque où les pétitions et manifestes pullulent, exigeant des intellectuels d'assumer cette responsabilité qu'ils revendiquent - au risque de l'inflation de prise de parti. Même Clamence parle de ses « confrères parisiens » qui «n'hésiteraient pas à lancer deux ou trois manifestes, peut-être même plus! Réflexion faite, j'ajouterais ma signature à la leur » (Camus, 1997 : 49), finit-il par décider. Camus, lui, en signe beaucoup trop, du point de vue de la droite. La gauche lui reproche de s'engager trop peu.

En 1948, il refuse de signer, en faveur de l'écrivain Jean Genet, menacé de la prison à perpétuité pour récidive de vol, la pétition que Sartre, Cocteau et d'autres adressent au Président de la République (Walker, 1994:23). Peut-on le comparer au refus de Paul Éluard dans le cas de Kalandra, deux ans plus tard?

En 1933, l'année cruciale de la montée du nazisme, le même Éluard et ses amis poètes surréalistes avaient élu pour muse et chanté Violette Nozière, une jeune parricide qui accusait son père d'inceste. Dans le chapitre "Surréalisme et révolution" de L'homme révolté, Camus critique ce choix d'idole, de même que le choix idéologique borné du surréaliste Éluard devenu communiste. Il n'est pas sans intérêt de constater que, cette même année 1933, la France avait suivi aussi une autre affaire criminelle - l'horrible meurtre perpétré par deux servantes, les sœurs Papin, sur leurs maitresses, fait divers tenu pour l'inspiration lointaine des Bonnes de Jean Genet.

Toujours est-il qu'en 1952, au moment de la polémique et des ruptures provoquées par la publication de L'homme révolté, Sartre publie Saint Genet comédien et martyr qui, dans l'opinion de certains (cf. Walker, 1993), aura également sensiblement influé sur La chute. À la fin de son essai de près de 700 pages, Sartre appelle Genet « un des héros de ce temps ». Camus, Sartre, Éluard, Genet, Kalandra - autant de faces d'un héros flou et changeant dans le « vestibule des lâches »?

\section{Égalité, fraternité ?}

Il ne faut tout de même pas oublier que La Chute avait son contre-poids prévu en Le premier homme, roman solaire et anti-proustien, une sorte de recherche du temps perdu par ceux qui n'ont pas de mémoire. Le roman pesant de tout son poids d' inachevé aux côtés de son auteur au moment de mourir - la mort faisant l' impossible justice à l' amitié, que ce soit dans l' hommage posthume rendu à Camus par Sartre ou, dans le poème de Paul Celan, par le «Nous » rêvé dans l' au-delà, de Kalandra innommé avec son ami Éluard.

Et si l'on veut adopter la logique de la poésie, des noms significatifs et des mots sous les mots saussuro-starobinskiens, le jour de l' accident mortel de Camus, le 4 janvier 1960, le romancier avait près de lui aussi une ombre pâle de Kalandra sous forme de calandre, à en croire Le Monde de l' époque :

C'est vers $14 \mathrm{~h} 15$ que s'est produit sur la route nationale numéro 5, à vingt quatre kilomètres environ de Sens, entre Champigny sur Yonne et Villeneuve la Guyard, l'accident qui a coûté la vie à Albert Camus. La voiture, une Facel Vega, se dirigeait vers Paris. L'écrivain était à l'avant, à côté du conducteur M. Michel Gallimard. D' après les premiers témoignages, la puissante automobile qui roulait à une très vive allure - 130 kilomètres à l'heure selon certains - a brusquement quitté le 
milieu de la route, toute droite à cet endroit, pour s'écraser contre un arbre à droite de la chaussée. Sous la violence du choc la voiture s'est disloquée. Une partie du moteur a été retrouvée à gauche de la route, à une vingtaine de mètres, avec la calandre et les phares. Des débris du tableau de bord et des portières ont été projetés dans les champs dans un rayon d'une trentaine de mètres. Le châssis s'est tordu contre l'arbre. D' après les premières constatations de la gendarmerie, l'accident aurait été provoqué par l'éclatement d'un pneu gauche, mais cette version n'est pas encore confirmée. Il n'est pas impossible que le conducteur ait eu un malaise. ${ }^{19}$ shoah, se donne la mort en se jetant dans la Seine, du haut du pont Mirabeau (celui d'Apollinaire) - ou du pont des Arts, celui du rire qui poursuivait Clamence, on ne sait. Dans sa poche, deux billets inutilisés pour une représentation d'En attendant Godot

Nous autres, enfants du début de millénaire, nous pouvons avoir le sommeil tranquille : munis de téléphones de plus en plus intelligents, nous n'avons plus à nous exécuter, il suffit d'appeler, tout pont parisien étant muni de plaques avec les numéros utiles pour le «Secours en cas de noyade ». Pour signer une pétition, on n’a qu’à cliquer. Pour celui qui se noie cependant, l'eau est restée toujours aussi froide. Par chance.

\section{BIBLIOGRAPHIE}

CAMUS, Albert (1962). L’Homme révolté. Paris : Gallimard, « Idées ».

CAMUS, Albert (1989). Carnets III (mars 1951 - décembre 1959). Paris : Gallimard.

CAMUS, Albert (1997). La chute. Paris: Gallimard, « Folio ».

CAMUS, Albert (2000). Zápisníky III (březen 1951 - prosinec 1959). Praha : Mladá fronta.

CELAN, Paul (1991). De seuil en seuil, traduit de l'allemand par Valérie Briet, édition bilingue.

Paris : Christian Bourgois Éditeur, « Détroits ».

DOLEŽAlovÁ, Markéta (2008-2013). « Záviš Kalandra (1902-1950) ». Praha : Ústav pro studium totalitních režimů [disponible le 21/05/2013] <URL : http://www.ustrcr.cz/cs/zavis-kalandra>.

DUFKOVÁ, Vlasta (2006). « Slovo o Kalandách », A2 kulturní čtrnáctideník, nº 39, p. 27.

DUFKovÁ, Vlasta (2014). « Les Kalendes tchèques », in Judit Maár, Krisztina Horváth (orgs.). Camus de l'autre côté du Mur : Réceptions de l'œuvre camusienne. Paris : L' Harmattan, Cahiers de la Nouvelle Europe, Collection du Centre Interuniversitaire d' Études Hongroises et Finlandaises de l' Université Sorbonne Nouvelle - Paris 3, nº 19, pp. 37-46.

LÉVI-VALENSI, Jacqueline (1996). «La chute » d'Albert Camus. Paris : Gallimard, « Folio ».

MÉLUSINE (2009). « Tracts surréalistes, Tome II (de février 1940 à avril 1960) ». Paris : Le Centre de Recherches sur le Surréalisme de Paris III [disponible le 10/09/2013] <URL : http://melusine.univparis3.fr/Tracts_surr_2009/Tracts_2_2009.htm>. 
Walker, David H. (1994). « Le criminel chez Camus », in David H. Walker (org.). Albert Camus, les extrêmes et l'équilibre : actes du colloque de Keele, 25-27 mars 1993. Amsterdam - Atlanta, GA : Editions Rodopi B.V., pp. 17-32.

Wikipédia, l'encyclopédie libre (2014). « Épuration à la Libération en France » [disponible le 20/01/2014] <URL : http://fr.wikipedia.org/w/index.php?title=\%C3\%89puration_\%C3\%A0_la_Lib \%C3\%A9ration_en_France\&oldid=100337403>.

\section{NOTES}

1. Cf. Dufková, "Les Kalandes tchèques »: version approfondie et documentée d'un premier article écrit en tchèque et paru en revue à Prague en 2006. Les actes du colloque ont été publiés en 2014.

2. Je souligne.

3. Je souligne.

4. Cf. Paul Éluard, Capitale de la douleur (1926).

5. Je souligne.

6. Cf. «Et par le pouvoir d'un mot / Je recommence ma vie » in Paul Éluard, « Liberté ».

7. $C f$. « Télégramme au Président de la République Tchécoslovaque » (Combat, 17-18 juin 1950) in Mélusine, 2009.

8. André Breton, « Lettre ouverte à Paul Eluard» (Combat, 13 juin 1950). Ibidem.

9. Publié dans l' Action, 1950. Cf. ibid., note (1). Je souligne.

10. $C f$. « La révolution pour les surréalistes n'était pas une fin qu'on réalise au jour le jour, dans l'action, mais un mythe absolu et consolateur. Elle était 'la vie véritable, comme l'amour', dont parlait Éluard, qui n'imaginait pas que son ami Kalandra dût mourir de cette vie-là. »

11. Sans pourtant le détourner, en 1956, de son retour au parti communiste dont il avait été exclu en 1950, et le serait une seconde fois, en 1970.

12. Je souligne.

13. Je souligne.

14. C'est moi qui divise en « vers » la prose de Camus.

15. Cf. Fonds de la Bibliothèque Nationale de Prague. (Apud Doležalová, 2008-2013).

16. Roman écrit en 1921-1923 et resté inachevé.

17. Texte intégral : «Le talon de notre brave GPU vient d'écraser un nid de vipères venimeuses ! Fini le ravage de ces reptiles abjects! Les trotskistes-terroristes-nihilistes enfin confondus ! Le dépravé sans la moindre trace d'un quelconque sentiment patriotique, le bandit Záviš Kalandra finit par avouer! Sous le poids des documents accusateurs dont une peinture documentaire de son crime bestial, et dans l'ambiance de vérité où l'on peut, aujourd'hui, respirer librement, il s'effondra pour avouer qu'il avait assassiné Přemysl Otakar II à Marchfeld ! Il se tenait à la tête d'un centre vertical collaborant avec Milota de Dědice [le noble tenu -injustement, selon les historiens - pour traître de son roi dans ladite bataille] afin de détacher les pays alpins de la Couronne de Bohême! Le procureur général transmettra le texte détaillé de la sentence au tribunal public!»

18. Je souligne.

19. Le Monde du 6 janvier 1960. Je souligne. 


\section{RÉSUMÉS}

Partant d'un pastiche du poème «Liberté » de Paul Éluard, dans les Carnets III d' Albert Camus, contemporains de l'écriture de La chute dont un des titres provisoires, lermontovien, avait été Un héros de notre temps, l'article se propose de déceler la possible répercussion, dans ce dernier récit publié de Camus, de l'attitude du poète de la Résistance lors du procès truqué de son ami tchèque d'avant-guerre Záviš Kalandra, condamné à mort et exécuté en 1950. L'auteur de l'article se pose la question plus générale de la responsabilité de l'intellectuel et de son engagement civique dans le contexte des temps modernes de l'immédiat après-guerre.

The current article is based on a pastiche of Paul Éluard's poem "Liberty" in Albert Camus's Notebooks III when Camus was working on the last of his larger prose texts published, i.e. The Fall, and one of the provisory titles of which bore a Lermontovian borrowing of A Hero of Our Time. The author of the article attempts to disclose in Camus's novel a possible projection of French resistance poet Éluard's reaction to the Czechoslovak show trial with his pre-WWII Czech friend Záviš Kalandra, sentenced on trumped-up charges and executed in 1950, and poses a more general question on the civic responsibility and engagement of the intellectual vis-à-vis the period following the end of WWII.

INDEX

Mots-clés : Camus (Albert), Éluard (Paul), Kalandra (Záviš), procès politiques, engagement civique

Keywords : Camus (Albert), Éluard (Paul), Kalandra (Záviš), political trials, civic engagement

\section{AUTEUR}

\section{VLASTA DUFKOVÁ}

Un. Charles de Prague

vlasta.dufkova[at]ff.cuni.cz 